\title{
EXPLORANDO LA INFLUENCIA DE LOS ROLES DE BELBIN EN LA CALIDAD DEL DISEÑO LÓGICO DE UNA BASE DE DATOS GENERADO POR ESTUDIANTES DE INGENIERÍA DE SOFTWARE
}

Raúl A. Aguilar Vera ${ }^{1}$

Adriana Peña Pérez Negrón ${ }^{2}$

adriana.pena@cucei.udg.mx

Julio C. Díaz ${ }^{1}$

julio.diaz@correo.uady.mx

Juan P. Ucán Pech ${ }^{1 *}$

juan.ucan@correo.uady.mx

${ }^{1}$ Facultad de Matemáticas, Universidad Autónoma de Yucatán,Mérida, Yucatán, México.

${ }^{2}$ Centro Universitario de Ciencias Exactas e Ingenierías, Universidad de Guadalajara Guadalajara, Jalisco, México

*Autor para Correspondencia

\section{RESUMEN}

En este artículo se presenta un estudio exploratorio en el que se analiza la influencia de los Roles de Belbin en actividades de desarrollo de software, particularmente, el Diseño Lógico de una Base de Datos; dicha actividad es considerada como un proceso de abstracción que consiste en transformar la representación de la Base de Datos vinculada a un problema, de un Modelo de Entidad Relación (Modelo Conceptual) a un Modelo Relacional (Modelo lógico). Las variables consideradas en el estudio fueron la Calidad del diseño y el Esfuerzo en la tarea. En virtud de ser un primer estudio (exploratorio), se utilizó una muestra basada en estudiantes avanzados de Ingeniería de Software. En el caso de la Calidad, los resultados permiten identificar diferencias significativas con el rol MonitorEvaluador respecto de los otros seis roles, obteniendo éste un mejor grado de Calidad; en el caso de la variable Esfuerzo, no se identificaron diferencias entre tratamientos.

\section{PALABRAS CLAVE}

Experimentación, Diseño de Bases de Datos, Ingeniería de Software. 


\title{
EXPLORING THE INFLUENCE OF BELBIN ROLES ON THE QUALITY OF THE LOGICAL DESIGN OF A DATABASE GENERATED BY SOFTWARE ENGINEERING STUDENTS
}

\begin{abstract}
This paper presents an exploratory study that analyzes the influence of Belbin Roles in software development activities, particularly, the Logical Design of a Database; this activity is considered as an abstraction process that consists of transforming the representation of the Database linked to a problem, from a Relationship Entity Model (Conceptual Model) to a Relational Model (Logical Model). The variables considered in the study were the Quality of the design and the Effort in the task. By virtue of being a first study (exploratory), a sample based on advanced students of Software Engineering was used. In the case of Quality, the results allow to identify significant differences with the Monitor-Evaluator role with respect to the other six roles, obtaining a better grade of Quality; In the case of the Effort variable, no differences were identified between treatments.
\end{abstract}

\section{KEYWORDS}

Database Design, Experimentation, Software Engineering.

\section{INTRODUCCIÓN}

A medio siglo de la denominada "Crisis del Software", la Ingeniería de Software (IS) dispone en la actualidad de un cuerpo de conocimientos aceptado en lo general por profesionistas e investigadores de la aún joven disciplina (Bourque \& Fairley, 2014), no obstante, la dinámica de mejora en cuanto a calidad, tanto en sus procesos, como en los artefactos generados a través de los mismos, sigue siendo fuente de investigación para dicha disciplina ingenieril (Miramontes et al, 2016; Norambuena y Vega, 2017).

El estudio de los enfoques vinculados con los procesos de desarrollo, operación, mantenimiento y gestión del software, ha sido analizado con base en la relación de diversas variables, no obstante, el aspecto social intrínseco de la disciplina (Juristo \& Moreno, 2001), ha llevado a considerar al factor humano, como un aspecto de singular interés para su investigación. En (Morales y Vega, 2018) se propone un catálogo de factores humanos críticos para el éxito de propuestas de mejora al proceso software, entre dichos factores se encuentra el rol que desempeña un Ingeniero de Software al interior del equipo de trabajo. En torno a dicho factor, Acuña, Juristo \& Moreno (2006) caracterizan —mediante rasgos de personalidad - a los individuos para su asignación a los roles identificados en los equipos de desarrollo software, y utilizan la información del recurso humano de la organización en su conjunto, para identificar necesidades de entrenamiento.

El presente estudio, tiene como propósito explorar si los roles propuestos por Belbin (Belbin, 1993) tienen algún tipo de incidencia en tareas vinculadas con procesos software, en particular, en el diseño lógico generado para una base de datos, así como en el esfuerzo en la tarea.

\section{ANTECEDENTES}

Entre las investigaciones sobre los roles que desempeñan los integrantes de un equipo, existe hoy día diferenciación entre roles orientados a la actividad individual, es decir, que se encuentran descritos en función de la tarea que pueden desempeñar y los roles orientados al desempeño del equipo, es decir, se encuentran definidos en función de su comportamiento con sus pares al interior del equipo; este segundo tipo de rol, es conocido como rol de equipo y al parecer, su ausencia o presencia, tiene una influencia significativa en los logros del equipo (Aritzeta, Swailes \& Senior, 2005). 
De entre los estudios sobre roles de equipo, la propuesta de roles de Belbin (Bebin, 1981; Bebin, 1993) es sin lugar a dudas la más conocida entre consultores e investigadores, sobre todo, porque ofrece mecanismos para la identificación del rol primario que un individuo puede asumir en un equipo de trabajo, en función de su comportamiento, así como una propuesta sobre equilibrio y compatibilidad entre roles, al interior de un equipo de trabajo. En el ámbito de la IS, existen diversos estudios que se sustentan en la teoría de roles de Belbin para explorar mejoras tanto en los procesos de desarrollo, como en los productos generados con dichos procesos (Schoenhoff, 2001); la mayoría de los estudios utilizan como factor la integración de los equipos de desarrollo, considerando como alternativa proceso controlado- equipos con roles compatibles (Pollock, 2009; Abdulrahman et al, 2017; Aguilar et al, 2020). Un segundo conjunto de trabajos, con menor evidencia al respecto, gira en torno al desempeño de los roles en tareas individuales (Henry \& Stevens, 1999; Estrada y Peña, 2013).

\section{DISEÑO LÓGICO DE BASES DE DATOS}

El proceso de Diseño de una Base de Datos, como parte del desarrollo de un sistema de información, generalmente es concebido como un proceso de abstracción con diferentes niveles de representación, de acuerdo con (De Miguel, Piattini y Marcos, 1999) el proceso más consensuado es concebido en tres etapas y por tanto, tres niveles de representación: Diseño Conceptual, Diseño Lógico y Diseño Físico.

El diseño de una base de datos tiene su origen en las necesidades de datos especificadas en la fase de requisitos, lo cuales son transformados en un primer nivel de abstracción, en un Modelo Conceptual -Diseño Conceptual- en el que se representen los recursos de información de la organización con independencia de usuarios y/o aplicaciones en particular; el modelo de datos más recurrido para dicha abstracción, es el Modelo de Entidad/Relación, dicho modelo está basado en una percepción del mundo real consistente en objetos básicos llamados entidades y de relaciones entre estos objetos (Silberschatz, Korth \& Sudarshan, 2020).

El segundo nivel de abstracción, tiene como objetivo transformar el Modelo Conceptual en un segundo nivel de abstracción, adaptándolo al modelo de datos en el que se apoye el Sistema de Gestión de Base de Datos (SGBD) que se vaya a utilizar para implementación -Diseño Lógico. Durante el siglo XX los sistemas relacionales dominaron el mercado y por ello el Modelo Relacional ha sido utilizado como modelo por excelencia para esta segunda etapa del proceso; dicho modelo es sin lugar a dudas el fundamento de la tecnología moderna de base de datos (Date, 2004), se ocupa de tres aspectos principales de la información: la estructura de datos, la manipulación de datos y la integridad de los datos; se basa en la teoría matemática de las relaciones, y los datos se estructuran lógicamente en forma de relaciones, comúnmente denominadas tablas. Debido a que el Modelo de Entidad/Relación y el Modelo Relacional comparten los mismos principios básicos de diseño, es posible aplicar un conjunto de reglas de derivación que permiten la transformación del Modelo Conceptual al Modelo Lógico Relacional (Silberschatz, Korth \& Sudarshan, 2020).

Finalmente, el Diseño Físico tiene como propósito la implementación, lo más eficiente posible, del modelo lógico; para dicho proceso, se utiliza el sub-lenguaje de definición de datos del SGBD.

\section{PLANIFICACIÓN DEL EXPERIMENTO CONTROLADO}

Una de las metodologías empíricas más recurridas en el ámbito de la IS, es la experimentación, particularmente, la experimentación en entornos controlados (Basili, Selby \& Hutchens, 1996); dicha metodología nos ayuda a identificar y en su caso, comprender las posibles relaciones entre factores y variables dependientes, ambos parámetros inmersos en quehacer del proceso software. Entre los elementos característicos de los estudios controlados encontrados en la literatura, se destaca el uso de grupos de estudiantes como sujetos experimentales; en (Genero, Cruz-Lemus y Piattini, 2014) los autores indican que dicha muestra académica permite al investigador obtener evidencia preliminar para confirmar o refutar hipótesis que pueden ser contrastadas posteriormente en contextos industriales.

El presente estudio tiene como propósito explorar, mediante la ejecución de un experimento controlado, la influencia de los roles de Belbin en la Calidad del Diseño Lógico de una Base de Datos, generado por estudiantes de Ingeniería de Software — previamente caracterizados con los roles de Belbin; así como en el Esfuerzo en la tarea. 


\subsection{Factor y Tratamientos}

El factor considerado en el estudio es definido como el rol desempeñado por el sujeto en la tarea de Diseño Lógico de una Base de Datos, particularmente, se identifican nueve tratamientos, los cuales se corresponden con los nueve roles propuestos por Belbin (ver tabla 1).

Tabla 1. Caracterización de los Roles de Belbin

\begin{tabular}{|c|c|c|}
\hline Tipo & Rol & Caracteristicas \\
\hline \multirow{3}{*}{ Acción } & $\begin{array}{l}\text { Impulsor } \\
\text { (Sharper: } \boldsymbol{S} \text { ) }\end{array}$ & $\begin{array}{l}\text { Retador, dinámico, trabaja bien bajo presión. Tiene iniciativa y } \\
\text { coraje para superar obstáculos. }\end{array}$ \\
\hline & $\begin{array}{l}\text { Implementador } \\
\text { (Implementer: } \mathbf{I})\end{array}$ & $\begin{array}{l}\text { Disciplinado, leal, conservador y eficiente. Transforma las ideas } \\
\text { en acciones. }\end{array}$ \\
\hline & $\begin{array}{c}\text { Finalizador } \\
\text { (Completer-Finisher: } \mathbf{C F})\end{array}$ & $\begin{array}{l}\text { Esmerado, concienzudo, ansioso. Busca los emrores y las } \\
\text { omisiones. Realiza las tareas en el plazo establecido. }\end{array}$ \\
\hline \multirow{3}{*}{ Mental } & $\begin{array}{l}\text { Cerebro } \\
\text { (Plant: } \boldsymbol{P})\end{array}$ & $\begin{array}{l}\text { Creativo, imaginativo, poco ortodoxo. Resuelve problemas } \\
\text { dificiles. }\end{array}$ \\
\hline & $\begin{array}{c}\text { Monitor Evaluador } \\
\text { (Monitor-Evaluator: } \mathbf{M E} \text { ) }\end{array}$ & $\begin{array}{l}\text { Serio, perspicaz y estratega. Percibe todas las opciones. Juzga } \\
\text { con exactitud. }\end{array}$ \\
\hline & $\begin{array}{c}\text { Especialista } \\
\text { (Specialist: } S P \text { ) }\end{array}$ & $\begin{array}{l}\text { Solo le interesa una cosa a un tiempo, cumplidor del deber. } \\
\text { Aporta cualidades y conocimientos especificos. }\end{array}$ \\
\hline \multirow{3}{*}{ Social } & $\begin{array}{l}\text { Coordinador } \\
\text { (Chairman: } \mathrm{CH} \text { ) }\end{array}$ & $\begin{array}{l}\text { Maduro, seguro de si mismo. Aclara las metas. Tiene iniciativa } \\
\text { y coraje para superar obstáculos. }\end{array}$ \\
\hline & $\begin{array}{l}\text { Investigador de Recursos } \\
\text { (Resouce Investigator: } R I \text { ) }\end{array}$ & $\begin{array}{l}\text { Extrovertido, entusiasta comunicativo. Busca nuevas } \\
\text { oportunidades. Desarrolla contactos. }\end{array}$ \\
\hline & $\begin{array}{l}\text { Cohesionador } \\
\text { (Teamworker: }: \mathrm{W} \text { ) }\end{array}$ & $\begin{array}{l}\text { Cooperador, apacible, perceptivo y diplomático. Escucha y evita } \\
\text { enfrentamientos. }\end{array}$ \\
\hline
\end{tabular}

\subsection{Hipótesis y Variables}

El primer par de hipótesis, utiliza como variable dependiente, una métrica vinculada con la Calidad del Diseño Lógico de la Base de Datos (DL-BD) generado por los estudiantes de IS.

- Ho1: Las medias respecto de la calidad del DL-BD generadas por cada uno de los roles, no presentan diferencias.

- $\mathrm{H}_{11}$ : Las medias respecto de la calidad del DL-BD generadas por cada uno de los roles, difiere en al menos un par de éstos.

El segundo par de hipótesis, utiliza como variable dependiente, el Esfuerzo utilizado por los estudiantes de IS, para concluir la tarea de generar el Diseño Lógico de la Base de Datos (DL-BD).

- Ho2: El esfuerzo registrado para generar el DL-BD por cada uno de los roles, no presenta diferencias significativas.

- $\mathrm{H}_{12}$ : El esfuerzo registrado para generar el DL-BD por cada uno de los roles, presenta diferencias significativas en al menos un par de éstos.

\subsection{Métricas consideradas en el estudio}

Para evaluar la calidad del DL-BD, seleccionamos como factor la Correctitud (McCall et al, 1977), entendido ésta como:

- Correctitud: El DL-BD se corresponde con las especificaciones del artefacto que le precede, en este caso, el Diseño Conceptual de la Base de Datos.

En virtud de que dicho factor es difícil de identificar y ante la falta de terminología estándar, hemos seleccionado como métrica, la Completitud, entendida ésta como:

- Completitud: Grado en el que el DL-BD generado por el sujeto experimental, es completo en comparación con los elemento del Diseño Lógico Correcto (DLC).

Operacionalmente, la Completitud (CO) será medida en función de un promedio entre los indicadores de: Relaciones o Tablas (IRE), Claves primarias (ICP) y Vínculos entre Relaciones (IVR), tal y como se especifica en las fórmulas I, II, II y IV, que se especifican a continuación: 


$$
\begin{aligned}
& \mathrm{CO}=(\mathrm{IRE}+\mathrm{ICP}+\mathrm{IVR}) / 3 \\
& \mathrm{IRE}=\frac{\sum_{i=1}^{n} \frac{\left(\sum_{j=1}^{m} x i j\right)}{m}}{n} \\
& \text { Donde Xij es un valor dicotómico que representa la posesión o no } \\
& \text { del atributo } \mathrm{j} \text { de la tabla } \mathrm{i} \text {, de acuerdo con las } \mathrm{n} \text { tablas del DLC } \\
& \mathrm{ICP}=\frac{\sum_{i=1}^{p} Y i}{p} \\
& \begin{array}{l}
\text { Donde Yi es un valor dicotómico que representa la posesión o no } \\
\text { de la clave primaria de la tabla i, de acuerdo con las p claves el DLC }
\end{array} \\
& \operatorname{IVR}=\frac{\sum_{i=1}^{q} Z i}{q}
\end{aligned}
$$

En cuanto a la segunda variable dependiente, el Esfuerzo, operacionalmente se considerará el Tiempo Medio - en minutos - utilizado por un estudiante para concluir la tarea, en este caso, se obtendrá mediante la diferencia entre la hora de finalización y la hora de inicio.

\subsection{Diseño Experimental}

El diseño experimental que resulta más apropiado para nuestro estudio, es el diseño factorial con una fuente de variación, y nueve tratamientos para las dos variables dependientes (ver tabla 2).

Tabla 2. Diseño Experimental

\begin{tabular}{|c|c|c|}
\hline Factor & Tratamiento & Variable Dependiente \\
\hline Rol & $\begin{array}{c}\text { Impulsor, Implementador, } \\
\text { Finalizador, Cerebro, } \\
\text { Monitor-Evaluador, Especialista, } \\
\text { Coordinador, Investigador de Recursos } \\
\text { y Cohesionador }\end{array}$ & $\begin{array}{c}\text { Calidad } \\
\text { Esfuerzo }\end{array}$ \\
\hline
\end{tabular}

\subsection{Propuesta de Análisis Estadístico}

Para el análisis de los datos, se generará una primera sección de estadística descriptiva con tablas de resumen para los valores obtenidos, así como gráficos de caja y bigotes para analizar visualmente el comportamiento de los mismos.

En el caso del análisis inferencial para las variables dependientes Calidad y Esfuerzo, elegimos el análisis de varianza de una vía, debido a que nos permite realizar pruebas de hipótesis para determinar si existe 0 no diferencias significativas entre las medias de los valores recogidos en la variable dependiente, para los diferentes tratamientos. El análisis de la varianza (Gutiérrez y De la Vara, 2012) es una técnica que permite construir con los datos, un modelo estadístico que describe el impacto de un solo factor categórico sobre una variable dependiente, el modelo lineal asociado es el siguiente:

$$
Y_{i j}=\mu+\beta_{i}+\varepsilon_{i j}
$$

donde Yij es la ij-ésima medición, $\mu$ es un parámetro común para todos los tratamientos, denominado media global, $\beta i$ es un parámetro que mide el efecto del i-ésimo tratamiento, y eij es el error sistemático atribuible a la medición Yij. 


\section{EJECUCIÓN DEL EXPERIMENTO}

Para el análisis de los datos, se generará una primera sección de estadística descriptiva con tablas de resumen para los valores obtenidos, así como gráficos de caja y bigotes para analizar visualmente el comportamiento de los mismos.

\subsection{Partcicipantes / Sujetos Experimentales}

La muestra por conveniencia estuvo conformada por 33 estudiantes que cursaban la asignatura Experimentación en Ingeniería de Software, ubicada en el séptimo semestre del Plan de Estudios vigente de la carrera antes citada. A los 33 participantes se les administró el instrumento de autopercepción propuesto por Belbin(1981), con el fin de identificar el rol primario que cada estudiante podría desempeñar; cabe destacar que dicho instrumento no incluye al rol especialista, y en el caso de la muestra, ningún estudiante estuvo identificado con el rol de investigador de recursos.

La tabla 3 presenta la muestra total de los 33 sujetos experimentales distribuidos en los siete tratamientos resultantes; para el análisis de la variable Calidad; tres productos fueron descartados en virtud de que los archivos digitales no resultaban claros para su análisis, así mismo, para la variable Esfuerzo, dos sujetos no registraron el tiempo de finalización de la tarea.

Tabla 3. Muestra Experimental

\begin{tabular}{|c|c|c|c|}
\hline \multirow{2}{*}{ Rol } & \multicolumn{3}{|c|}{ Muestra (\#) } \\
\cline { 2 - 4 } & Total & Calidad & Esfuerzo \\
\hline Cerebro & 2 & 2 & 2 \\
\hline Cohesionador & 4 & 3 & 4 \\
\hline Coordinador & 4 & 4 & 2 \\
\hline Finalizador & 8 & 7 & 8 \\
\hline Implementador & 5 & 4 & 5 \\
\hline Impulsor & 5 & 5 & 5 \\
\hline Monitor Evaluador & 5 & 5 & 5 \\
\hline Total & $\mathbf{3 3}$ & $\mathbf{3 0}$ & $\mathbf{3 1}$ \\
\hline
\end{tabular}

\subsection{Sesión Experimental}

Para el experimento, el profesor - autor principal- realizó en una sesión previa a la sesión experimental, un repaso del tema vinculado al Diseño Lógico de Bases de Datos, tema analizado en una asignatura del sexto semestre denominada Diseño de Bases de Datos.

Durante la sesión experimental, el profesor entregó el Diseño Conceptual de una Base de Datos (figura 1), aclaró dudas del Modelo, y solicitó que registren la hora de inicio de la tarea, también proporcionó instrucciones para que los sujetos registren el tiempo de finalización, y posteriormente digitalicen y suban a la plataforma el Modelo Lógico generado. 


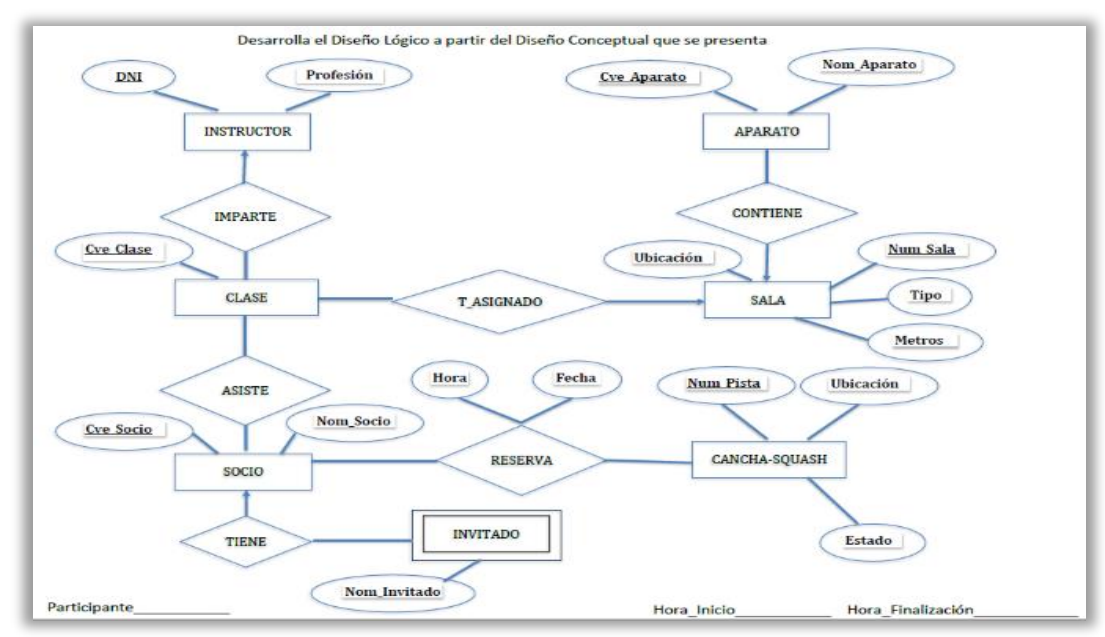

Figura 1. Diseño Conceptual de la BD proporcionado

Al final de la sesión, los sujetos entregaron el modelo lógico indicando los datos de identificación (ver figura 2), siendo dichos documentos, los objetos experimentales utilizados en la fase del análisis experimental.

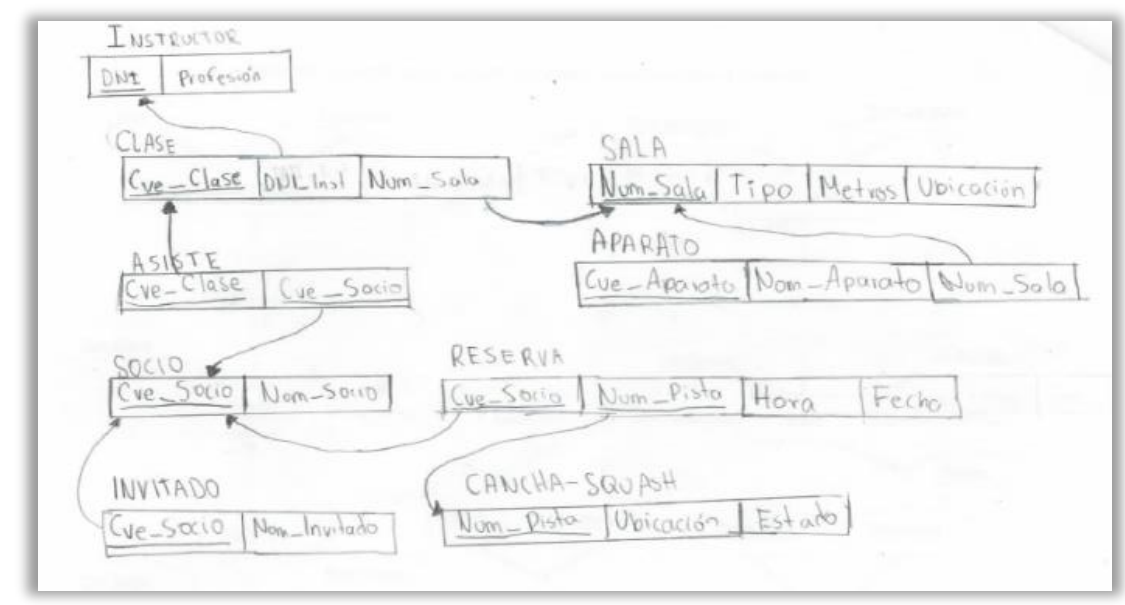

Figura 2. Ejemplo de Objeto experimental (Diseño lógico de la BD)

\section{ANÁLISIS EXPERIMENTAL}

En esta sección se presenta el análisis estadístico descriptivo e inferencial, para las dos variables dependientes, con base en los datos obtenidos en el experimento controlado.

\subsection{Análisis Descriptivo}

La Tabla 4 presenta algunas de las medidas más importantes de tendencia central y variabilidad para la variable Calidad; podemos observar que el rol Monitor-Evaluador presenta la media más elevada, y después del rol Cerebro, es el segundo tratamiento con menor variabilidad y el tercero con menor rango; cabe mencionar que ambos roles son clasificados como de tipo mental. 
Tabla 4. Resumen estadístico de la variable Calidad

\begin{tabular}{|c|c|c|c|c|c|c|}
\hline Rol & $\#$ & $\mu$ & O & Min & Max & Rango \\
\hline Cerebro & 2 & 0.6489 & 0.0272 & 0.6296 & 0.6682 & 0.03858 \\
\hline Cohesionador & 3 & 0.6857 & 0.1406 & 0.5555 & 0.8348 & 0.2793 \\
\hline Coordinador & 4 & 0.7295 & 0.1030 & 0.6296 & 0.8379 & 0.2083 \\
\hline Finalizador & 7 & 0.6920 & 0.1235 & 0.6172 & 0.9629 & 0.3456 \\
\hline Implementador & 4 & 0.6172 & 0.0400 & 0.5740 & 0.6667 & 0.0925 \\
\hline Impulsor & 5 & 0.7574 & 0.1483 & 0.5925 & 0.9583 & 0.3657 \\
\hline Monitor Evaluador & 5 & 0.9484 & 0.0369 & 0.9027 & 1.0 & 0.0972 \\
\hline
\end{tabular}

Para analizar visualmente el comportamiento de los datos, generamos un diagrama de caja y bigotes; dicho gráfico nos permite observar la dispersión y la simetría de los conjuntos de datos para cada uno de los tratamientos, así como la comparación entre los mismos; en la figura 3 podemos observar que el comportamiento desfasado de los datos del tratamiento Monitor-Evaluador, esto nos induce pensar que posiblemente exista una diferencia con los otros seis tratamientos.

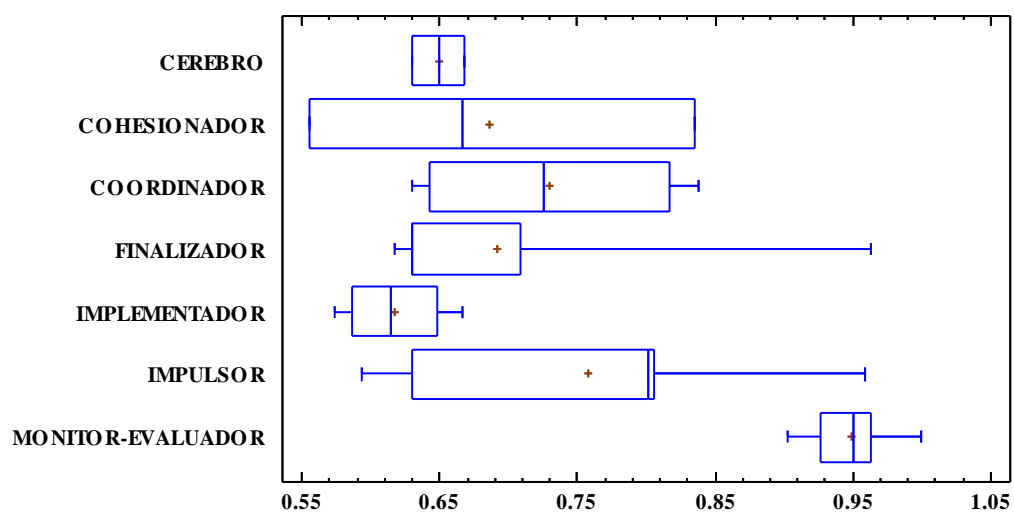

Figura 3. Diagrama de Caja y Bigotes para la variable Calidad

En la Tabla 5 se listan algunas de las medidas de tendencia central y variabilidad más importantes para la variable Esfuerzo; podemos observar que los roles de tipo social - Cohesionador y Coordinador- son los que presentaron mayor esfuerzo en tarea, siendo el Coordinador el rol con menor variabilidad de entre los siete tratamientos. Observamos también que el mayor tiempo destinado para la tarea ocupó apenas una tercera parte del tempo planificado para la tarea -30 minutos. 
Tabla 5. Resumen estadístico de la variable Esfuerzo

\begin{tabular}{|c|c|c|c|c|c|c|}
\hline Rol & $\#$ & $\mu$ & O & Min & Max & Rango \\
\hline Cerebro & 2 & 14.0 & 5.6568 & 10.0 & 18.0 & 8.0 \\
\hline Cohesionador & 4 & 22.75 & 5.4390 & 17.0 & 30.0 & 13.0 \\
\hline Coordinador & 2 & 20.0 & 1.4142 & 19.0 & 21.0 & 2.0 \\
\hline Finalizador & 8 & 19.375 & 4.8384 & 12.0 & 28.0 & 16.0 \\
\hline Implementador & 5 & 15.6 & 3.5071 & 11.0 & 20.0 & 9.0 \\
\hline Impulsor & 5 & 15.4 & 4.8270 & 11.0 & 22.0 & 11.0 \\
\hline Monitor Evaluador & 5 & 19.4 & 3.0495 & 15.0 & 23.0 & 8.0 \\
\hline
\end{tabular}

Al igual que con la variable Calidad, generamos un diagrama de caja y bigotes para la variable Esfuerzo (ver figura 4); no observamos comportamientos que nos induzcan a pensar en diferencias entre tratamientos.

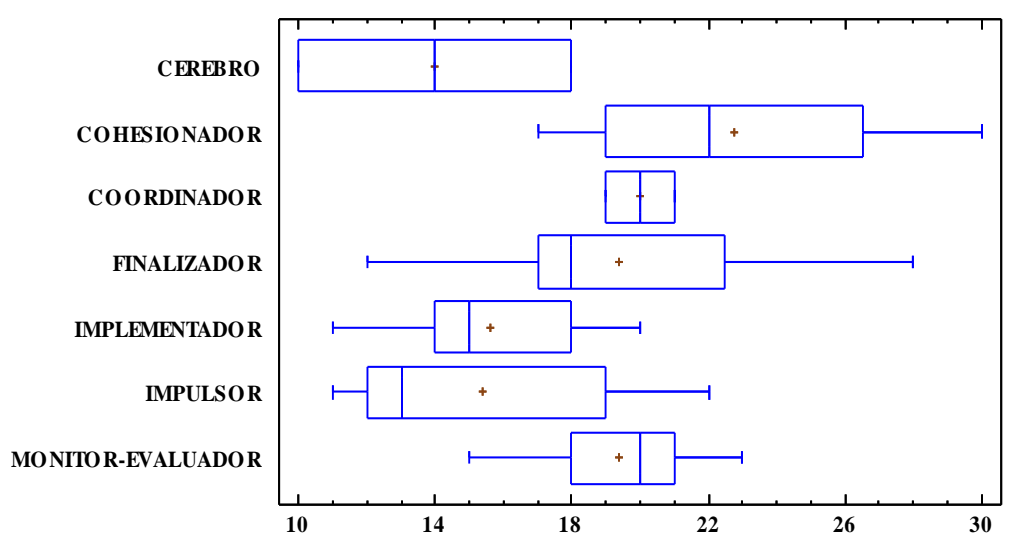

Figura 4. Diagrama de Caja y Bigotes para la variable Esfuerzo

\subsection{Análisis Inferencial}

Con el propósito de evaluar estadísticamente las diferencias entre los tratamientos de las variables Calidad y Esfuerzo, se aplicó el Análisis de Varianza de una vía; el resultado de evaluar con el ANOVA se ilustra en la tabla 6.

Tabla 6. ANOVA para las variables Calidad y Esfuerzo

\begin{tabular}{|c|c|c|}
\hline Variable & Razón F & Valor de p \\
\hline Calidad & 4.69 & $0.0030^{*}$ \\
\hline Esfuerzo & 1.85 & 0.1314 \\
\hline
\end{tabular}

En el caso de la variable Calidad, el valor $\mathrm{p}$ de la prueba $\mathrm{F}$ es menor que 0.05 por lo cual, las hipótesis de nulidad $\mathrm{H} 01$ puede ser rechazada, es decir, podríamos afirmar que existe al menos un par de tratamientos que presentan diferencias estadísticamente significativas entre sus medias, con un $5 \%$ de significancia. 
Para identificar cuáles de los tratamientos son diferentes, generamos el gráfico de medias con intervalos de confianza de acurdo con la prueba LSD, la cual se ilustra en la figura 5; dicho gráfico nos permite hacer una comparación visual y estadística de las medias de los tratamientos. Como podemos observar, al parecer el único tratamiento que presenta un desfase respecto de los otros seis tratamientos, es el correspondiente al rol Monitor-Evaluador.

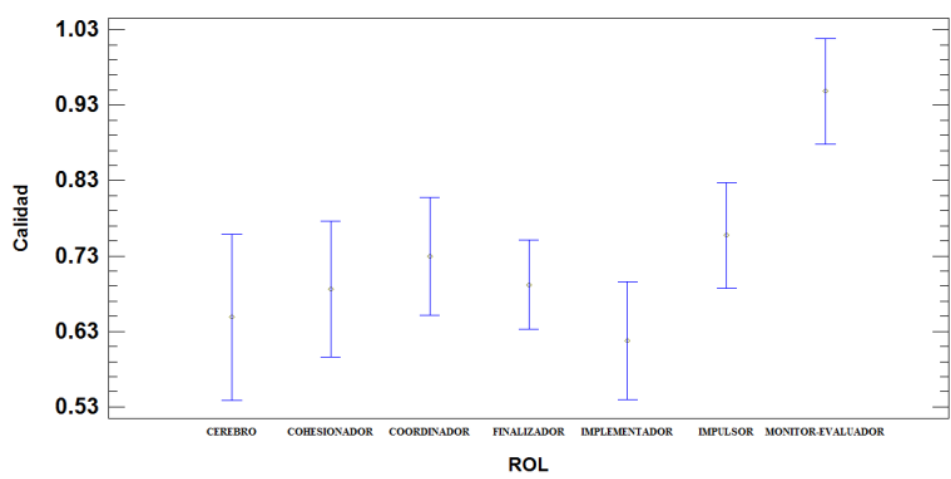

Figura 5. Gráfico de Medias (Método LSD) para la variable Calidad

La prueba de múltiples rangos para la variable calidad, mediante el método LSD, nos arroja diferencias entre 6 parejas de tratamientos tal y como se ilustra en la taba 7; dicho análisis coincide con lo considerado con el análisis visual de la figura 5 .

Tabla 7. Prueba de múltiples rangos para la variable Calidad

\begin{tabular}{|c|c|c|c|}
\hline Contraste & Sig. & Diferencia & +/-Límites \\
\hline Cerebro - Monitor-Evaluador & $*$ & -0.299537 & 0.184751 \\
\hline Cohesionador - Monitor-Evaluador & $*$ & -0.262757 & 0.161264 \\
\hline Coordinador - Monitor-Evaluador & $*$ & -0.218904 & 0.14813 \\
\hline Finalizador - Monitor-Evaluador & $*$ & -0.256437 & 0.129299 \\
\hline Implementador - Monitor-Evaluador & $*$ & -0.331173 & 0.14813 \\
\hline Impulsor - Monitor-Evaluador & $*$ & -0.191049 & 0.139658 \\
\hline
\end{tabular}

En el caso de la variable Esfuerzo, el valor $\mathrm{p}$ de la prueba $\mathrm{F}$ es mayor que 0.05 por lo cual, deberíamos rechazar la hipótesis de nulidad $\mathrm{H} 02$, es decir, podemos afirmar que no existe evidencia, al menos con un $5 \%$ de significancia, de diferencias entre las medias de los tratamientos.

\subsection{Validación de Modelos}

Es importante mencionar que el modelo ANOVA tiene asociado tres supuestos que es necesario validar antes de utilizar la información que nos ofrece: (1) Los errores experimentales de sus datos se distribuyen normalmente, (2) No existe diferencia entre la varianza de los tratamientos y (3) existe independencia entre las muestras. Para validar el primer supuesto, usaremos el gráfico de probabilidad normal de residuos; dicha técnica permite evaluar visualmente su comportamiento en función de la distribución normal. 


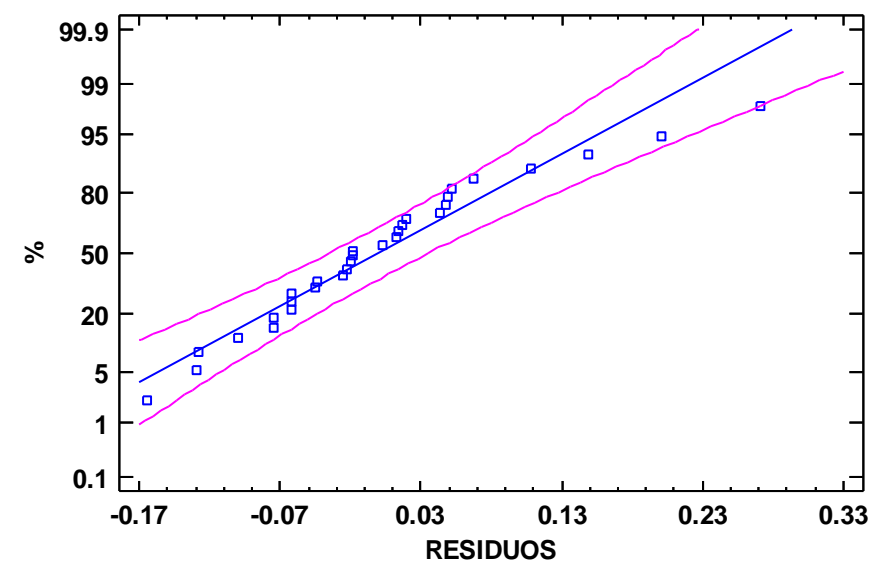

Figura 6. Gráfico de probabilidad normal para la variable Calidad

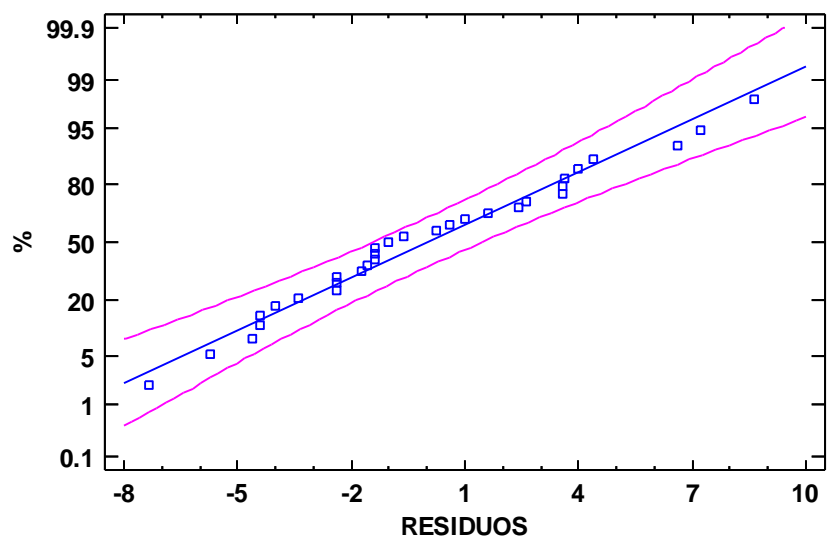

Figura 7. Gráfico de probabilidad normal para la variable Esfuerzo

Como se puede ver en las gráficas que se ilustran en las figuras 6 y 7 , en ambas variables, Calidad y Esfuerzo, respectivamente, los puntos no muestran desviaciones de la diagonal, por lo que es posible asumir que los residuos tienen una distribución normal.

Por su parte, la prueba de Levene permite evaluar diferencias significativas entre las varianzas de los dos conjuntos de datos (homocedasticidad); en la tabla 8 podemos observar que en ambas variables (Calidad y Esfuerzo) el $p$ valor es mayor que 0.05, lo cual nos indica que no existe una diferencia estadísticamente significativa entre las desviaciones estándar de los tratamientos, con un nivel del $95.0 \%$ de confianza.

Tabla 7. Prueba de Levene (Calidad y Esfuerzo)

\begin{tabular}{|c|c|c|}
\hline Variable & Prueba & Valor de p \\
\hline Calidad & 1.78903 & 0.1459 \\
\hline Esfuerzo & 0.784262 & 0.5907 \\
\hline
\end{tabular}

Para validar el supuesto de independencia de los datos, generamos un gráfico de residuales versus secuencia, para cada variable (ver figuras 8 y 9 ). 


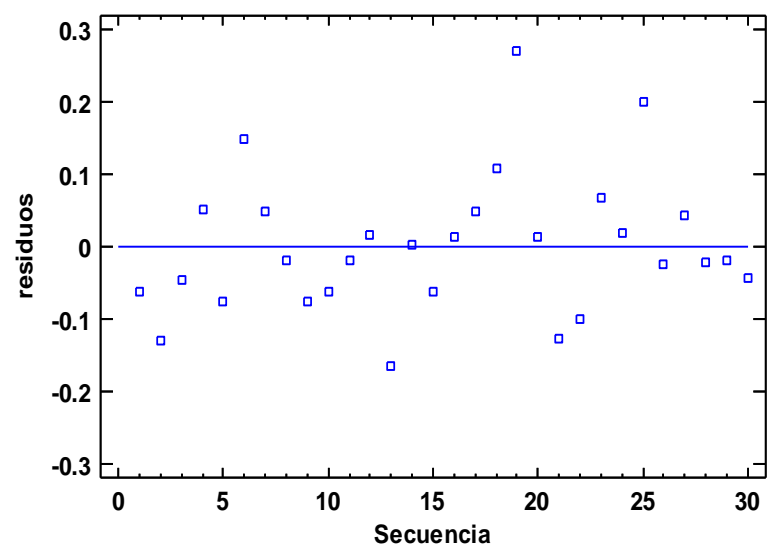

Figura 8. Gráfico de residuos vs. secuencia para la variable Calidad

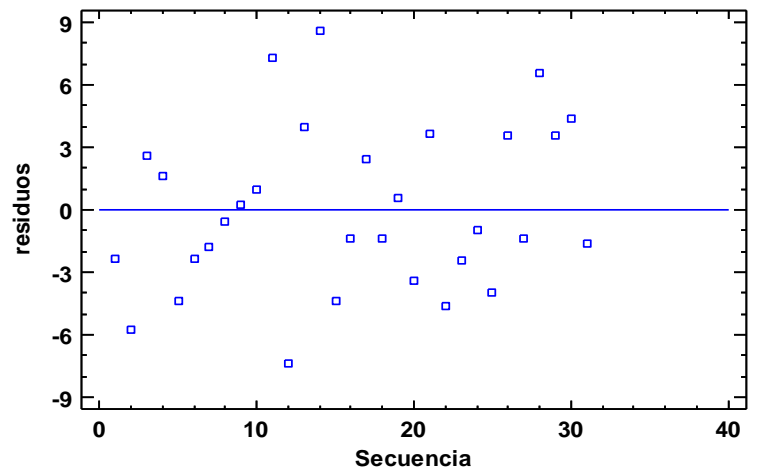

Figura 9. Gráfico de residuos vs. secuencia para la variable Esfuerzo

Podemos observar en ambas gráficos - figuras 8 y 9 - que no existe una tendencia en los datos, por lo cual, es posible suponer, que los datos provienen de poblaciones independientes.

Superados los tres supuestos del modelo ANOVA para ambas variables, podemos afirmar que los comentarios derivados de la tabla 6 , son válidos.

\section{CONCLUSIONES}

En este estudio presentamos un primer experimento controlado con el propósito de explorar si el uso de la teoría de roles de Belbin puede ser benéfico para identificar a los Ingenieros de Software con mejor habilidad en la tarea de Diseño Lógico de una Base de Datos.

Con el experimento controlado, encontramos que con la variable vinculada al producto -Calidad del Diseño Lógico de la BD- el rol Monitor-Evaluador presenta diferencias significativas, habiendo obtenido un grado de calidad mejor que el de los otros seis roles participantes en el experimento; el rol Monitor-Evaluador es uno de los dos roles de tipo mental identificados en el experimento; dicho resultado coincide parcialmente con los resultados reportados en (Henry \& Stevens, 1999) en el sentido de que es uno de los tres roles identificados -Especialista, Monitor-Evaluador y Finalizador - con una buena aportación en la tarea de diseño; también coincide con lo comentado en (Schoenhoff, 2001), en torno a que es uno de los dos roles - Cerebro y Monitor-Evaluador-que presentan atracción por la fase de diseño. 
Por su parte, para la variable esfuerzo en la tarea, los roles no mostraron diferencias significativas, posiblemente la tarea diseñada no fue lo suficientemente compleja para requerir mayores tiempos de dedicación, en la que se pudiesen observar diferencias entre los roles participantes.

Con lo obtenido en este estudio, los autores proponen continuar con el desarrollo de experimentos controlados, en tareas vinculadas con el proceso software, a fin de generar conocimiento en torno a posibles relaciones entre tipos de tareas y roles con un mejor desempeño en las mismas.

\section{AGRADECIMIENTOS}

Agradecemos el apoyo brindado por la Secretaría de Educación Pública (México) a través del proyecto P/PROFEXE-2020-31MSU0098J-13, así como a los alumnos de la Licenciatura en Ingeniería de Software de la UADY que participaron en el experimento.

\section{REFERENCIAS}

Abdulrahman, A., Omar, M., Ahmad, M. \& Ahmed, V. (2017). An Analysis of Belbin Team Roles in Software Engineering Team. Journal of engineering and Applied Sciences, 12(Special Issue 5), pp. 6878-6883.

Acuña, S., Juristo, N. \& Moreno, A. (2006). Emphasizing Human Capabilities in Software Development. IEEE Software, 23(2), pp.94-101.

Aguilar, R., Muñoz, M., Díaz, J. y Ucán, J. (2020). Explorando la influencia de los roles de Belbin en la especificación de requisitos de software. Revista Ibérica de Sistemas y Tecnologías de la Información. (36), pp. 34-49.

Aritzeta, A., Swailes,S. \& Senior, B. (2005). Team roles: Psychometric evidence, construct validity and team building. Hull, UK: University Hull.

Basili, V., Selby, R. \& Hutchens, D. (1996). Experimentation in Software Engineering. IEEE Transactions on Software Engineering, 12(7), pp. 733-743.

Belbin, M. (1993). Team roles at Work. Oxford, USA: Elsevier Butterworth Heinemann.

Belbin, M. (1981). Management teams: Why they succeed or fail. New York, USA: John Wiley \& Sons.

Bourque, P \& Fairley, R. (Eds.) (2014). Guide to the Software Engineering Body of Knowledge (SWEBOK V3.0). IEEE Computer Society.

Date, C.J. (2004). An introduction to database systems. USA: Pearson Education

De Miguel, A., Piattini, M. y Marcos, E. (1999). Diseño de Bases de Datos Relacionales. España: RaMa.

Estrada, E. y Peña, A. (2013). Influencia de los roles de equipo en las actividades del desarrollador de software. Revista Electrónica de Computación, Informática, Biomédica y Electrónica. (1), pp.1-19.

Genero, M., Cruz-Lemus, J. y Piattini, M. (2014). Métodos de investigación en ingeniería de software. España: Ra-Ma.

Gutiérrez, H. y De la Vara, R. (2012). Análisis y Diseño de Experimentos (3ª ed.). Ciudad de México, México: McGraw Hill.

Henry, S. \& Stevens, K. (1999). Using Belbin's leadership role to improve team effectiveness: An empirical investigation. The Journal of Systems and Software, 44, pp. 241-250.

Juristo, N. \& Moreno, A. (2001). Basics of Software Engineering Experimentation. Boston, USA: Kluwer Academic Publishers.

McCall, J. A., Richards, P. K. \& Walters, G. F. (1977). Factors in Software Quality, Volumes I, II, and III. US Rome Air Development Center Reports US Department of Commerce USA. 
Miramontes, J., Muñoz, M. Calvo-Manzano, J. y Corona, B. (2017). Establecimiento del estado del arte sobre el aligeramiento de procesos de software. Revista lbérica de Sistemas y Tecnologías de Información, (17), pp. 16-25.

Morales, N. y Vega, V. (2018). Factores Humanos y la Mejora de Procesos de Software. Propuesta inicial de un catálogo que guíe su gestión. Revista lbérica de Sistemas y Tecnologías de Información, (29), pp. 30-42.

Norambuena, B. y Vega, V. (2017). Minería de procesos de software: una revisión de experiencias de aplicación. Revista Ibérica de Sistemas y Tecnologías de Información, (21), pp. 51-66.

Pollock, M. (2009). Investigating the relationship between team role diversity and team performance in information systems teams. Journal of Information Technology Management, vol. 20(1), pp .42-55.

Schoenhoff, P.K. (2001). Belbin's Company Worker, The Self-Perception Inventory and Their Application to Software Engineering Teams (Master Of Science Thesis). USA: Virginia Polytechnic Institute and SU.

Silberschatz, A, Korth, H. \&Sudarshan, S. (2020). Database Systems Concepts. 7th. ed. USA: McGrawHill. 

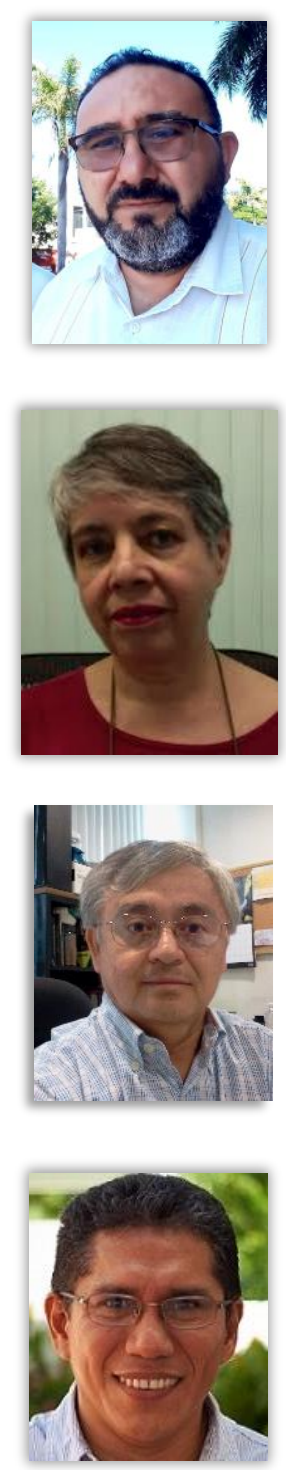

Raúl Antonio Aguilar Vera obtuvo el grado de Doctor por la Universidad Politécnica de Madrid, España (Mención de Doctor Europeo) y el de Master en Ingeniería de Software por la misma Institución, posee también el grado de Maestro en Educación Superior por la Universidad Autónoma de Yucatán. Actualmente es profesor en la Facultad de Matemáticas de la Universidad Autónoma de Yucatán. Es coordinador de la Sección Académica de Ingeniería de Software de la Academia Mexicana de Computación (AMEXCOMP). Su trabajo de investigación incluye las áreas de Ingeniería de Software e Informática Educativa.

Adriana Peña Pérez Negrón es doctora en Ingeniería de la Informática de la Universidad Politécnica de Madrid, España. Realizó una estancia investigación en la University of Salford, Reino Unido para la mención de "Doctor Europeo" Actualmente es profesor investigador en el Departamento de Ciencias Computacionales del CUCEI de la Universidad de Guadalajara, México. Es responsable del Cuerpo Académico Informática Aplicada del Programa para el Desarrollo Profesional Docente. Sus principales líneas de investigación son la Realidad Virtual y el estudio del comportamiento en medios digitales

Julio Cesar Díaz Mendoza es Ingeniero Industrial en Producción por el Instituto Tecnológico de Mérida (ITM). Maestro en Tecnologías de Información, por la Universidad Interamericana para el Desarrollo (UNID). Especialista en Docencia de la Universidad Autónoma de Yucatán. Actualmente es profesor en la Facultad de Matemáticas de la Universidad Autónoma de Yucatán, e imparte asignaturas en las carreras de Ingeniería de Software y Ciencias de la Computación. Su interés se enfoca al área de Ingeniería de Software Educativo.

Juan Pablo Ucán Pech es Doctor en Sistemas Computacionales por la Universidad del Sur, campus Mérida, México. Maestro en Sistemas Computacionales con especialidad en Ingeniería de Software por el Instituto Tecnológico de Mérida, México. Actualmente es profesor en la Facultad de Matemáticas de la Universidad Autónoma de Yucatán. Es miembro de la Academia Mexicana de Computación (AMEXCOMP). Su trabajo de investigación se centra en temas relacionados con la Ingeniería de Software, Ingeniería Web e Informática Educativa.

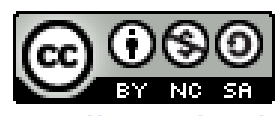

Esta obra está bajo una licencia de Creative Commons Reconocimiento-NoComercial-Compartirlgual 2.5 México. 